\title{
Necessary and sufficient global optimality conditions for NLP reformulations of linear SDP problems
}

\author{
L. Grippo*, L. Palagi*, V Piccialli* \\ * Sapienza Università di Roma \\ Dipartimento di Informatica e Sistemistica "A. Ruberti" \\ Via Ariosto 25 - 00185 Roma - Italy \\ e-mail (Grippo): grippo@dis.uniroma1.it \\ e-mail (Palagi): palagi@dis.uniroma1.it \\ e-mail (Piccialli): piccialli@dis.uniroma1.it
}

\begin{abstract}
In this paper we consider the standard linear SDP problem, and its low rank nonlinear programming reformulation, based on a Gramian representation of a positive semidefinite matrix. For this nonconvex quadratic problem with quadratic equality constraints, we give necessary and sufficient conditions of global optimality expressed in terms of the Lagrangian function.
\end{abstract}

Keywords: semidefinite programming - low-rank factorization - optimality conditions. 


\section{Introduction}

The standard linear SDP problem we consider is of the form:

$$
\begin{aligned}
\min & \operatorname{trace}(Q X) \\
& \operatorname{trace}\left(A_{i} X\right)=b_{i}, \quad i=1, \ldots, m \\
& X \succeq 0, \quad X \in \mathcal{S}^{n},
\end{aligned}
$$

where the data matrices $\mathrm{Q}$ and $A_{i}$ for $i=1, \ldots, m$ are $n \times n$ real symmetric matrices, trace $(Q X)$ denotes the trace-inner product of matrices, and the $n \times n$ matrix variable $\mathrm{X}$ is required to be symmetric and positive semidefinite, as indicated with the notation $X \succeq 0, X \in \mathcal{S}^{n}$, where $\mathcal{S}^{n}$ is the space of real $n \times n$ symmetric matrices.

This class of problems contains important problems as special cases, such as linear or quadratic programming, and arises in a wide variety of applications in system and control theory, combinatorial optimization, approximation theory, robust optimization, and mechanical and electrical engineering.

Among the main approaches for solving linear SDP problems are interior point methods (see for example the survey [10] and references therein), and first order non linear programming methods ([2]). Interior point methods are in general able to solve SDP problems of small or medium size with high accuracy, and are proved to converge in polynomial time to an $\epsilon$ optimal solution. However, in practice, the dimension of the problem that can be solved is still limited to a maximum of a few thousand variables for the most efficient codes. First order nonlinear programming methods work efficiently in practice and can solve much larger problems, but convergence to a global solution is not guaranteed. We are interested in this class of methods, and the aim of this paper is to give a theoretical result that further justifies this approach.

Burer and Monteiro in $[2,3]$ recast a general linear SDP problem as a low rank semidefinite programming problem (LRSDP) by applying the change of variables $X=R R^{T}$, where $R$ is a $n \times r, r<n$, rectangular matrix. They get the following problem

$$
\begin{aligned}
\min & \operatorname{trace}\left(Q R R^{T}\right) \\
& \operatorname{trace}\left(A_{i} R R^{T}\right)=b_{i}, \quad i=1, \ldots, m \\
& R \in \mathbb{R}^{n \times r}, r \leq n .
\end{aligned}
$$

The value of $r$ is chosen by exploiting the result proved in Barvinok [1] and Pataki [12], that states that, under suitable assumptions, there exists an optimal solution of a linearly constrained SDP problem with rank $r$ satisfying $r(r+1) \leq m$, where $m$ is the number of linear constraints. Problem (1) is a nonlinear programming problem; although it has been 
proved $[1,12,2]$ that a value of $r$ exists such that there is a one-to one correspondence between global solutions of Problem (1) and global solutions of Problem (SDP), Problem (1) is a non convex problem, so that recognizing a global solution is a difficult task.

In this paper, under suitable assumptions on Problem (SDP), we state necessary and sufficient global optimality conditions in terms of the Lagrangian function. Similar conditions have already been proved to be sufficient in [3]. These conditions extend the necessary and sufficient ones proved in [5] for the special case of the semidefinite relaxation of the max cut problem and can be related to the necessary and sufficient global optimality conditions established for some classes of nonconvex quadratic problems (see e.g. $[6,11,9]$ ).

\section{Low rank SDP formulations}

It has been proved in $[2,5]$ that if Problem (SDP) admits a solution $X^{*}$ or rank $r$, this can be found by solving Problem (1). Actually Problem (1) can be rewritten as a standard nonlinear programming problem, by setting $R=\left(v_{1} \ldots v_{n}\right)^{T}, v_{k} \in \mathbb{R}^{r}$ for $k=1, \ldots, n$ so that $\left(R R^{T}\right)_{i j}=$ $v_{i}^{T} v_{j}$ and we get:

$$
\begin{aligned}
\min & q_{r}(v):=\sum_{i=1}^{n} \sum_{j=1}^{n} q_{i j} v_{i}^{T} v_{j} \\
& \sum_{k=1}^{n} \sum_{j=1}^{n}\left(A_{i}\right)_{k j} v_{k}^{T} v_{j}=b_{i}, i=1, \ldots, m, v_{k} \in \mathbb{R}^{r}, k=1, \ldots, n .
\end{aligned}
$$

The following result proved in [1] and [12] provides a useful upper bound on the value of $r$, that can be easily computed.

Proposition 1 Suppose that the feasible set of Problem (SDP) has an extreme point. Then there exists an $X \in \mathcal{S}^{n}$ optimal solution of (SDP) with rank $r$ satisfying the inequality

$$
r(r+1) / 2 \leq m
$$

This result implies that $r \leq \widehat{r}$ where

$$
\widehat{r}=\max \{k \in N: k(k+1) / 2 \leq m\}=\left\lfloor\frac{\sqrt{1+8 m}-1}{2}\right\rfloor .
$$

Therefore, for sufficiently large values of $r$, a global solution of Problem (2) gives a global solution of Problem (SDP). In particular, Problem (2) gives a global solution of Problem (SDP) for all $r \geq \widehat{r}$. 
We can recast Problem (2) in compact standard vector notation of nonlinear programming form by using Kronecker products $\otimes$ (see, for instance, [8]). We recall that given two matrices $A m \times n$ and $B p \times q$, the Kronecker product $A \otimes B$ is the $m p \times n q$ matrix given by

$$
A \otimes B=\left[\begin{array}{cccc}
a_{11} B & a_{12} B & \ldots & a_{1 n} B \\
\vdots & \vdots & \vdots & \vdots \\
a_{m 1} B & a_{n 2} B & \ldots & a_{m n} B
\end{array}\right] .
$$

Given a matrix $A \in \mathcal{S}^{n}$, with spectrum $\sigma(A)=\left\{\lambda_{1}, \ldots, \lambda_{n}\right\}$ and a matrix $B \in \mathcal{S}^{m}$, with spectrum $\sigma(B)=\left\{\mu_{1}, \ldots, \mu_{m}\right\}$, it is known (see [8]) that the spectrum of $A \otimes B$ is given by:

$$
\sigma(A \otimes B)=\left\{\lambda_{i} \mu_{j}: i=1, \ldots, n ; j=1, \ldots, m\right\} .
$$

Hence, letting $e_{i} \in \mathbb{R}^{n}$, we can write the vector $v \in \mathbb{R}^{n r}$ as

$$
v=\sum_{i=1}^{n}\left(e_{i} \otimes v_{i}\right)=\left(\begin{array}{c}
v_{1} \\
\vdots \\
v_{n}
\end{array}\right)
$$

so that we have

$$
v_{i}=\left(e_{i} \otimes I_{r}\right)^{T} v .
$$

Therefore we can write the objective function of Problem (2) as:

$$
\begin{aligned}
\sum_{i=1}^{n} \sum_{j=1}^{n} q_{i j} v_{i}^{T} v_{j} & =\sum_{i=1}^{n} \sum_{j=1}^{n} q_{i j}\left(\left(e_{i} \otimes I_{r}\right)^{T} v\right)^{T}\left(e_{j} \otimes I_{r}\right)^{T} v \\
& =\sum_{i=1}^{n} \sum_{j=1}^{n} v^{T}\left(q_{i j} e_{i} e_{j}^{T} \otimes I_{r}\right) v=v^{T}\left(Q \otimes I_{r}\right) v .
\end{aligned}
$$

With similar reasoning the constraints can be written as

$$
\sum_{k=1}^{n} \sum_{j=1}^{n}\left(A_{i}\right)_{k j} v_{k}^{T} v_{j}=v^{T}\left(A_{i} \otimes I_{r}\right) v
$$

Thus, we obtain the nonlinear programming problem

$$
\begin{aligned}
\min \quad v^{T}\left(Q \otimes I_{r}\right) v & =q_{r}(v) \\
v^{T}\left(A_{i} \otimes I_{r}\right) v & =b_{i} \quad i=1, \ldots, m,
\end{aligned}
$$

which is the problem we will focus on in the rest of the paper. 


\section{Optimality conditions}

We are interested in Problem $\left(\mathrm{NLP}_{r}\right)$ as an instrument to solve Problem (SDP), and hence we need to solve it to global optimality. However, since Problem $\left(\mathrm{NLP}_{r}\right)$ is a non convex problem, the best we can expect from a nonlinear programming algorithm is to produce a point satisfying some necessary optimality conditions. The question we want to answer is when a stationary point $\hat{v}$ of Problem $\left(\mathrm{NLP}_{r}\right)$ is a global minimizer that solves Problem (SDP). We remark that, from now on, for sake of simplicity, we adopt the following terminology: whenever we say that a point $v^{*} \in \mathbb{R}^{n r}$ solves Problem (SDP) we mean that $X^{*}=V^{*} V^{* T}$, where $V^{*}=\left(v_{1}^{*} \ldots v_{n}^{*}\right)^{T}$, is an optimal solution of Problem (SDP), namely

$$
q_{r}\left(v^{*}\right)=\operatorname{trace}\left(Q X^{*}\right)=z_{\mathrm{SDP}}^{*}
$$

We want to be able to recognize a global minimum point of Problem $\left(\mathrm{NLP}_{r}\right)$ for suitable values of $r$.

The Lagrangian function for Problem $\left(\mathrm{NLP}_{r}\right)$ is, for an arbitrary fixed value $r \geq 1$,

$$
\begin{aligned}
L(v, \lambda) & =v^{T}\left(Q \otimes I_{r}\right) v+\sum_{i=1}^{m} \lambda_{i}\left(v^{T}\left(A_{i} \otimes I_{r}\right) v-b_{i}\right) \\
& =v^{T}\left[\left(Q+\sum_{i=1}^{m} \lambda_{i} A_{i}\right) \otimes I_{r}\right] v-\lambda^{T} b
\end{aligned}
$$

where $\lambda=\left(\lambda_{1}, \ldots, \lambda_{n}\right)^{T}$.

Definition 1 (Stationary point of Problem $\left(\mathbf{N L P}_{r}\right)$ ) $A$ point $\hat{v} \in$ $\mathbb{R}^{n r}$ is a stationary point of Problem $\left(\mathrm{NLP}_{r}\right)$, if there exists a Lagrange multiplier $\hat{\lambda} \in \mathbb{R}^{m}$ such that $(\hat{v}, \hat{\lambda}) \in \mathbb{R}^{n r} \times \mathbb{R}^{m}$ satisfies:

$$
\begin{array}{ll}
{\left[\left(Q+\sum_{i=1}^{m} \lambda_{i} A_{i}\right) \otimes I_{r}\right] \hat{v}} & =0 \\
\hat{v}^{T}\left(A_{i} \otimes I_{r}\right) \hat{v} & =b_{i}, \quad i=1, \ldots, m .
\end{array}
$$

Moreover, $\hat{v} \in \mathbb{R}^{n r}$ is a second order stationary point of Problem $\left(\mathrm{NLP}_{r}\right)$, if there exists a Lagrange multiplier $\hat{\lambda} \in \mathbb{R}^{m}$ such that $(\hat{v}, \hat{\lambda}) \in \mathbb{R}^{n r} \times \mathbb{R}^{m}$ satisfies (5) and

$$
z^{T}\left[\left(Q+\sum_{i=1}^{m} \lambda_{i} A_{i}\right) \otimes I_{r}\right] z \geq 0
$$

for every $z \in \mathbb{R}^{n r}$ such that $\hat{v}^{T}\left(A_{i} \otimes I_{r}\right) z=0$ for $i=1, \ldots, m$. 
First we state some useful properties of the Lagrange multipliers at a stationary point deriving from the structure of Problem $\left(\mathrm{NLP}_{r}\right)$.

Proposition 2 Let $\hat{v} \in \mathbb{R}^{n r}$ be a stationary point of Problem $\left(\mathrm{NLP}_{r}\right)$, and let $\hat{\lambda} \in \mathbb{R}^{m}$ be the corresponding Lagrange multiplier. Then we have:

$$
\hat{\lambda}^{T} b=-q_{r}(\hat{v}) .
$$

Proof. Let $\hat{v}$ be a stationary pair of Problem $\left(\mathrm{NLP}_{r}\right)$ so that

$$
\frac{1}{2} \nabla_{v} L(\hat{v}, \hat{\lambda}) \equiv\left(Q \otimes I_{r}\right) \hat{v}+\sum_{i=1}^{m} \hat{\lambda}_{i}\left(A_{i} \otimes I_{r}\right) \hat{v}=0 .
$$

Premultiplying both sides of (7) by $\hat{v}^{T}$ we can write:

$$
\hat{v}^{T}\left(Q \otimes I_{r}\right) \hat{v}+\sum_{i=1}^{m} \hat{\lambda}_{i} \hat{v}^{T}\left(A_{i} \otimes I_{r}\right) \hat{v}=0 .
$$

Therefore, as $\hat{v}^{T}\left(A_{i} \otimes I_{r}\right) \hat{v}=b_{i}$, for all $i=\ldots, m$, we obtain

$$
\sum_{i=1}^{m} \hat{\lambda}_{i} b_{i}=-\hat{v}^{T}\left(Q \otimes I_{r}\right) \hat{v}
$$

which yields (6).

In order to exploit the information coming from the relationship between Problem $\left(\mathrm{NLP}_{r}\right)$ and Problem (SDP), we apply duality theory to the convex Problem (SDP) to get global optimality conditions for it.

Let $u \in \mathbb{R}^{m}$, we can write the standard Lagrangian dual of Problem (SDP):

$$
\begin{array}{ll}
\max & b^{T} u \\
& Q-\sum_{i=1}^{m} u_{i} A_{i} \succeq 0 .
\end{array}
$$

Denote by $u^{*} \in \mathbb{R}^{m}$ an optimal solution of Problem (8), and let

$$
b^{T} u^{*}=z_{\mathrm{DUAL}}^{*}
$$

We suppose that the following assumption is satisfied:

Assumption A1 Problem (SDP) and its dual have nonempty optimal solution sets with zero duality gap. 
Therefore, $X^{*}$ and $u^{*}$ are optimal solutions of the primal problem (SDP) and of its dual (8) respectively if and only if:

$$
\begin{aligned}
z_{\mathrm{SDP}}^{*}=\operatorname{trace}\left(Q X^{*}\right) & =b^{T} u^{*}=z_{\mathrm{DUAL}}^{*} \\
\operatorname{trace}\left(A_{i} X^{*}\right) & =b_{i} \quad i=1, \ldots, m \\
X^{*} & \succeq 0 \\
Q-\sum_{i=1}^{m} u_{i}^{*} A_{i} & \succeq 0 .
\end{aligned}
$$

By posing $u_{i}=-y_{i}$ for $i=1, \ldots, n$ we can write problem (8) as

$$
\begin{array}{ll}
\min \quad & b^{T} y \\
& Q+\sum_{i=1}^{m} y_{i} A_{i} \succeq 0,
\end{array}
$$

so that the primal dual optimality conditions can be rewritten as

$$
\begin{aligned}
\operatorname{trace}\left(Q X^{*}\right) & =-b^{T} y^{*} \\
\operatorname{trace}\left(A_{i} X^{*}\right) & =b_{i} \quad i=1, \ldots, m \\
X^{*} & \succeq 0 \\
Q+\sum_{i=1}^{m} y_{i}^{*} A_{i} & \succeq 0 .
\end{aligned}
$$

These necessary and sufficient optimality conditions require the solution of the dual problem, while we are interested in conditions on the Lagrangian of Problem $\left(\mathrm{NLP}_{r}\right)$. Indeed, the following necessary and sufficient global optimality condition can be proven.

Proposition 3 Suppose that Assumption A1 holds and that there exists an optimal solution $X^{*}$ of Problem (SDP) of rank $r$. Then a point $v^{*} \in$ $\mathbb{R}^{n r}$ is a global minimizer of Problem $\left(\mathrm{NLP}_{r}\right)$ if and only if there exists $a \lambda^{*} \in \mathbb{R}^{m}$ such that

$$
\begin{aligned}
& {\left[\left(Q+\sum_{i=1}^{m} \lambda_{i}^{*} A_{i}\right) \otimes I_{r}\right] v^{*}=0} \\
& Q+\sum_{i=1}^{m} \lambda_{i}^{*} A_{i} \succeq 0 \\
& v^{* T}\left(A_{i} \otimes I_{r}\right) v^{*}=b_{i}, \quad i=1, \ldots, m .
\end{aligned}
$$

Proof First assume that (11) are satisfied. By (6), we have $q_{r}\left(v^{*}\right)=$ $-b^{T} \lambda^{*}$. The vector $u^{*}=-\lambda^{*}$ is feasible for the dual problem (8), and hence $u^{*}$ is optimal for the dual. Therefore, the primal dual optimality 
conditions (9) and the assumption that there exists an optimal solution $X^{*}$ of Problem (SDP) of rank $r$ together give

$$
q_{r}^{*}=z_{\mathrm{SDP}}^{*}=z_{\mathrm{DUAL}}^{*}=-b^{T} \lambda^{*}=q_{r}\left(v^{*}\right) .
$$

As for the necessity part, we know that $v^{*}$ is a global minimum point of Problem $\left(\mathrm{NLP}_{r}\right)$ and that the corresponding $X^{*}=V^{*} V^{* T}$, where $V^{*}=\left(v_{1}^{*} \ldots v_{n}^{*}\right)^{T}$ is optimal for Problem (SDP). Therefore, we get by (10) that there exist $y^{*} \in \mathbb{R}^{n}$ such that

$$
\begin{aligned}
& -b^{T} y^{*}=q_{r}\left(v^{*}\right) \\
& Q+\sum_{i=1}^{m} y_{i}^{*} A_{i} \succeq 0,
\end{aligned}
$$

and hence:

$$
-b^{T} y^{*}=v^{* T}\left(Q \otimes I_{r}\right) v^{*} .
$$

Since $v^{* T}\left(A_{i} \otimes I_{r}\right) v^{*}=b_{i}, i=1, \ldots, m$, we can write

$$
b^{T} y^{*}=\sum_{i=1}^{m} y_{i}^{*} v^{* T}\left(A_{i} \otimes I_{r}\right) v^{*}=v^{* T}\left(\sum_{i=1}^{m} y_{i}^{*} A_{i} \otimes I_{r}\right) v^{*}
$$

that summed up with (12) gives

$$
v^{* T}\left[\left(Q+\sum_{i=1}^{m} y_{i}^{*} A_{i}\right) \otimes I_{r}\right] v^{*}=0 .
$$

Since $Q+\sum_{i=1}^{m} y_{i}^{*} A_{i}$ is positive semidefinite, by the properties of the Kronecker products, also the matrix $\left(Q+\sum_{i=1}^{m} y_{i}^{*} A_{i}\right) \otimes I_{r}$ is positive semidefinite, and hence we can represent it as the square of its square root so that (13) implies

$$
\left[\left(Q+\sum_{i=1}^{m} y_{i}^{*} A_{i}\right) \otimes I_{r}\right] v^{*}=0
$$

Therefore, $v^{*}$ is a stationary point of Problem $\left(\mathrm{NLP}_{r}\right)$ with corresponding Lagrange multiplier $y^{*}$, and setting $\lambda=y^{*}$ we have that (11) holds.

This proposition shows that the solution $u^{*}$ of the dual problem is actually obtained from the Lagrange multiplier $\lambda^{*}$ associated with the solution $v^{*}$ of Problem $\left(\mathrm{NLP}_{r}\right)$. We point out once more that problem $\left(\mathrm{NLP}_{r}\right)$ is a non convex optimization problem so that necessary and sufficient global optimality conditions are usually not available. In general, 
for non convex quadratic-quadratic minimization problems (quadratic objective function and quadratic constraints) global optimality conditions can be found in some particular situations (see $[6,11,9]$ ). A well known case is when there is one single quadratic constraint (equality or inequality), that is the case of the generalized trust region problem [11]. Most of these conditions consist essentially in requiring that the Hessian of the Lagrangian function is positive semidefinite at a stationary point, as in the above proposition. However, as far as we know, the result stated in Proposition 3 can not be derived from these previous results.

Note also that we do not require constraint qualifications on the quadratic problem $\left(\mathrm{NLP}_{r}\right)$, but we need Assumption A1 to be satisfied. It results that assuming that strong duality holds for Problem (SDP) is sufficient to ensure that the global minimum of Problem $\left(\mathrm{NLP}_{r}\right)$ is a stationary point, and this may explain why we do not need to impose constraint qualifications.

The condition stated in Proposition 3 can be computationally checked without solving the dual problem (8), since it requires only the knowledge of the Lagrange multiplier associated to the point $v^{*}$, which is provided by many algorithms that compute stationary points of nonlinear programming problems.

A different sufficient global optimality condition has been proved in [2] by Burer and Monteiro. In particular, for $r<n$ they prove the following result that gives a sufficient condition of global optimality.

Proposition 4 (Proposition 4 in [2]) Let $v^{*} \in \mathbb{R}^{n r}$, with $r<n$, be a local minimum point of Problem $\left(\mathrm{NLP}_{r}\right)$. Let $\hat{v} \in \mathbb{R}^{n(r+1)}$ be a point with components $\hat{v}_{i} \in \mathbb{R}^{r+1}$ such that

$$
\hat{v}_{i}=\left(\begin{array}{c}
v_{i}^{*} \\
0
\end{array}\right)
$$

If $\hat{v}$ is a local minimum of Problem $\left(\mathrm{NLP}_{r+1}\right)$, then $v^{*}$ is a global minimum point of Problem $\left(\mathrm{NLP}_{r}\right)$ that solves Problem (SDP).

Actually, by looking at the details of the proof of the above result in [2], it emerges that the only assumption needed is that $v^{*}$ and $\hat{v}$ are stationary points with the same Lagrange multiplier. This has been already observed in [5] for the special LRSDP deriving from the maxcut problem. Following the same reasoning we show that the condition of Proposition 4 is also necessary. By exploiting this result we can establish a new necessary and sufficient condition that can be computationally checked. Indeed, the following result holds. 
Proposition 5 Suppose that Assumption A1 holds and that there exists an optimal solution $X^{*}$ of Problem (SDP) of rank $r$. A point $v^{*} \in \mathbb{R}^{n r}$, with $r<n$, is a global minimum point of Problem $\left(\mathrm{NLP}_{r}\right)$ if and only if the following conditions hold:

(i) $v^{*}$ is a stationary point for Problem $\left(\mathrm{NLP}_{r}\right)$ with Lagrange multiplier $\lambda^{*} \in \mathbb{R}^{m}$

(ii) the point $\hat{v} \in \mathbb{R}^{n(r+1)}$ with components $\hat{v}_{i} \in \mathbb{R}^{r+1}$ defined as

$$
\hat{v}_{i}=\left(\begin{array}{c}
v_{i}^{*} \\
0
\end{array}\right)
$$

is a second order stationary point for Problem $\left(\mathrm{NLP}_{r+1}\right)$ with Lagrange multiplier $\lambda^{*} \in \mathbb{R}^{m}$.

Proof First of all, we prove sufficiency by rephrasing in our notation the proof given in [2]. For any $w=\left(w_{1}, \ldots, w_{n}\right)^{T} \in \mathbb{R}^{n}$, let us define the vector $z \in \mathbb{R}^{n r}$

$$
z^{T}=\left(\begin{array}{lllllll}
0_{r}^{T} & w_{1} & 0_{r}^{T} & w_{2} & \cdots & 0_{r}^{T} & w_{n}
\end{array}\right)
$$

which satisfies

$$
\hat{v}^{T}\left[A_{i} \otimes I_{r+1}\right] z=0, \quad \text { for all } i=1, \ldots, m .
$$

By the second order necessary conditions for Problem $\left(\mathrm{NLP}_{r+1}\right)$, we must have $z^{T}\left[\left(Q+\sum_{i=1}^{m} \lambda_{i}^{*} A_{i}\right) \otimes I_{r+1}\right] z \geq 0$ and therefore, by the expression of $z$, and setting $q_{i j}^{*}=\left(Q+\sum_{i=1}^{m} \lambda_{i}^{*} A_{i}\right)_{i j}$ we get

$$
\begin{aligned}
& 0 \leq z^{T}\left[\left(Q+\sum_{i=1}^{m} \lambda_{i}^{*} A_{i}\right) \otimes I_{r+1}\right] z= \\
& \left(\begin{array}{lllll}
0_{r}^{T} & w_{1} & \ldots & 0_{r}^{T} & \left.w_{n}\right)
\end{array}\right)\left(\begin{array}{c}
\sum_{j=1}^{n} 0_{r} q_{1 j}^{*} w_{j} \\
\vdots \\
0_{r} \\
\sum_{j=1}^{n} q_{n j}^{*} w_{j}
\end{array}\right) \\
& =\sum_{i=1}^{n} \sum_{j=1}^{n} q_{i j}^{*} w_{i} w_{j}=w^{T}\left(Q+\sum_{i=1}^{m} \lambda_{i}^{*} A_{i}\right) w
\end{aligned}
$$


where $w$ is any vector in $\mathbb{R}^{n}$, which implies $\left(Q+\sum_{i=1}^{m} \lambda_{i}^{*} A_{i}\right) \succeq 0$. Then the global optimality of $v^{*}$ follows from relation $(6)$, that says $q_{r}\left(v^{*}\right)=$ $-b^{T} \lambda^{*}$, and from the primal dual optimality conditions (10).

Now we prove the necessity part. Let $X^{*}$ be the global minimum solution of rank $r$ of Problem (SDP), and let $v^{*} \in \mathbb{R}^{n r}$ be the corresponding global solution of Problem $\left(\mathrm{NLP}_{r}\right)$. Define the vector $\hat{v} \in \mathbb{R}^{n(r+1)}$ with vector components given by (14), which is obviously feasible for Problem $\left(\mathrm{NLP}_{r+1}\right)$. We have that

$$
q_{r+1}(\hat{v})=q_{r}\left(v^{*}\right)=z_{\mathrm{SDP}}^{*}
$$

and hence $\hat{v}$ is a global minimum point of Problem $\left(\mathrm{NLP}_{r+1}\right)$. By Proposition 3 , it follows that there exists a $\lambda^{*} \in \mathbb{R}^{m}$ such that (11) hold. Therefore, by (11) and by the expression of $\hat{v}$, it follows

$$
\begin{aligned}
& {\left[\left(Q+\sum_{i=1}^{m} \lambda_{i}^{*} A_{i}\right) \otimes I_{r+1}\right] \hat{v}=} \\
& \left(\begin{array}{ccccc}
q_{11}^{*} I_{r} & 0_{r} & & q_{1 n}^{*} I_{r} & 0_{r} \\
0_{r}^{T} & q_{11}^{*} & \ldots & 0_{r}^{T} & q_{1 n}^{*} \\
\vdots & & \ddots & & \vdots \\
q_{n 1}^{*} I_{r} & 0_{r} & & q_{n n}^{*} I_{r} & 0_{r} \\
0_{r}^{T} & q_{n 1}^{*} & & 0_{r}^{T} & q_{n n}^{*}
\end{array}\right)\left(\begin{array}{c}
v_{1}^{*} \\
0 \\
\vdots \\
v_{n}^{*} \\
0
\end{array}\right)=\left(\begin{array}{c}
\sum_{j=1}^{n} q_{1 j}^{*} v_{j}^{*} \\
0 \\
\vdots \\
\sum_{j=1}^{n} q_{n j}^{*} v_{j}^{*} \\
0
\end{array}\right)=0 .
\end{aligned}
$$

Moreover, since by (11) we have that $Q+\sum_{i=1}^{m} \lambda_{i}^{*} A_{i} \succeq 0$, it follows from the properties of Kronecker products that $\left(Q+\sum_{i=1}^{m} \lambda_{i}^{*} A_{i} \succeq 0\right) \otimes I_{r+1} \succeq$ 0 , and hence we have proved that $v^{*}$ is a stationary point for Problem $\left(\mathrm{NLP}_{r}\right)$ with Lagrange multiplier $\lambda^{*}$, and $\hat{v}$ is a second order stationary point of Problem $\left(\mathrm{NLP}_{r+1}\right)$ with the same multiplier $\lambda^{*}$.

Remark The requirement that $v^{*}$ is a stationary point for Problem $\left(\mathrm{NLP}_{r}\right)$ with Lagrange multiplier $\lambda^{*}$, and $\hat{v}$ is a stationary point of Problem $\left(\mathrm{NLP}_{r+1}\right)$ with the same multiplier $\lambda^{*}$ is always verified if the Linear Independence Constraint Qualification (LICQ) is satisfied at $\hat{v}$ for problem $\left(\mathrm{NLP}_{r+1}\right)$. Indeed, in this case the Lagrange multiplier $\hat{\lambda}$ associated to $\hat{v}$ is unique. It follows from (17),

$$
\left[\left(Q+\sum_{i=1}^{m} \lambda_{i}^{*} A_{i}\right) \otimes I_{r+1}\right] \hat{v}=0,
$$

where $\lambda^{*}$ is the Lagrange multiplier associated to $v^{*}$. As the Lagrange multiplier associated to $\hat{v}$ is unique, then $\hat{\lambda}=\lambda^{*}$. Hence $\hat{v}$ is a stationary point of Problem $\left(\mathrm{NLP}_{r}\right)$ with Lagrange multiplier $\lambda^{*}$. 


\section{Concluding Remarks}

In this paper, we have described a new class of quadratic problems arising as nonlinear programming reformulations of linear SDP problems for which it is possible to state necessary and sufficient global optimality conditions. An algorithmic use of these conditions can be the subject of further investigation.

\section{References}

[1] A. Barvinok. Problems of distance geometry and convex properties of quadratic maps. Discrete Computational Geometry, 13:189-202 (1995).

[2] S. Burer and R.D.C. Monteiro. A nonlinear programming algorithm for solving semidefinite programs via low-rank factorization. Math. Programming, Ser. B 95:329-357 (2003).

[3] S. Burer and R.D.C. Monteiro. Local Minima and Convergence in Low-Rank Semidefinite Programming. Mathematical Programming, Ser. A, 103:427-444 (2005).

[4] M.X. Goemans and D.P. Williamson. Improved approximation algorithms for maximum cut and satisfiability problems using semidefinite programming. J. Assoc. Comput. Mach., 42(6):1115-1145 (1995).

[5] L. Grippo, L. Palagi, V. Piccialli An unconstrained minimization method for solving low rank SDP relaxations of the max cut problem. DIS Technical Report, 07-07, Sapienza Università di Roma (2007).

[6] J. B. Hiriart-Urruty, Conditions for Global Optimality 2. Journal of Global Optimization, 13:349-367 (1998).

[7] R. A. Horn and C. R. Johnson, Matrix analysis, Cambridge University Press, Cambridge, 1985.

[8] R. A. Horn and C. R. Johnson. Topics in matrix analysis, Cambridge University Press, New York, 1986.

[9] V. Jeyakumar, A. M. Rubinov and Z. Y. Wu. Non-convex quadratic minimization problems with quadratic constraints: global optimality conditions. Math. Program.. Ser. A, 110:521-541 (2007). 
[10] R. D. C. Monteiro.First- and second-order methods for semidefinite programming. Mathematical Programming 97:209-244 (2003).

[11] J. Moré, Generalization of the trust region problem, Optimization Methods and Software, 2 (1993), pp. 189-209.

[12] G. Pataki. On the rank of extreme matrices in semidefinite programs and the multiplicity of optimal eigenvalues. Mathematics of Operations Research, 23:339358 (1998). 